Meta

Journal des traducteurs

Translators' Journal

\title{
Index du volume 12
}

Volume 12, numéro 4, décembre 1967

Aspects de la terminologie

URI : https://id.erudit.org/iderudit/003219ar

DOI : https://doi.org/10.7202/003219ar

Aller au sommaire du numéro

Éditeur(s)

Les Presses de l'Université de Montréal

ISSN

0026-0452 (imprimé)

1492-1421 (numérique)

Découvrir la revue

Citer ce document

(1967). Index du volume 12. Meta, 12(4), 151-153.

https://doi.org/10.7202/003219ar

Ce document est protégé par la loi sur le droit d'auteur. L'utilisation des services d'Érudit (y compris la reproduction) est assujettie à sa politique d'utilisation que vous pouvez consulter en ligne.

https://apropos.erudit.org/fr/usagers/politique-dutilisation/
Cet article est diffusé et préservé par Érudit.

Érudit est un consortium interuniversitaire sans but lucratif composé de l’Université de Montréal, l'Université Laval et l'Université du Québec à Montréal. Il a pour mission la promotion et la valorisation de la recherche. https://www.erudit.org/fr/ 


\section{INDEX DU VOLUME 12}

\section{A. AUTEURS - AUTHORS}

1. Audette, Adrienne: "Au dossier de "nursing ", vol. $12, \mathrm{n}^{\circ} 3$, p. 88

2. Bernier, Normand: "Le français face à la civilisation scientifique et technique ", vol. 12 , $\mathrm{n}^{\circ}$ 1, p. 23.

"Société des Traducteurs et Interprètes du Canada ", vol. 12, no 1, p. 28.

"Table ronde sur la formation du traducteur», vol. 12, $\mathrm{n}^{0} 1, \mathrm{p} .29$.

"Chronique de la STIC », vol. 12, n 2, p. 67.

"Colloque sur la traduction automatique ", vol. 12, no 3, p. 103.

"Société des Traducteurs et Interprètes du Canada », vol. 12, n 3, p. 107.

"Association des Traducteurs et Interprètes de l'Ontario », vol. 12, $\mathrm{n}^{\circ}$ 4, p. 141.

"Société des Traducteurs du Québec ", vol. 12, n 4, p. 140.

3. Clas, André : « Défini, indéfini ", vol. $12, \mathrm{n}^{\circ} 2$, p. 39.

"À propos de "giggle", vol. 12, n 2, p. 56.

4. Claxton, Patricia D. : "Culture Vulture », vol. $12, \mathrm{n}^{\circ} 1$, p. 9.

5. Clément, Clémence : "Cercle des traducteurs", vol. $12, n^{\circ} 2$, p. 68.

"Cercle des Traducteurs », vol. 12, n 3, p. 105.

6. Comité de linguistique de Radio-Canada: "Consultation", vol. 12, no 3, p. 91.

7. Connolly, Jean-Guy : "Cercle des Traducteurs", vol. 12, no 1, p. 33.

8. Côté, Yves-Aubert: "Brève réflexion sur la terminologie comptable", vol. 12, no 4 , p. 127.

9. Darbelnet, Jean : "Portrait du vocabulaire français", vol, $12, \mathrm{n}^{\circ} 1$, p. 14.

"Structure et information ", vol. 12, $\mathrm{n}^{\circ} 2, \mathrm{p} .58$.

"Ressources néologiques du français contemporain ", vol. 12, $\mathrm{n}^{\circ} 4$, p. 111.

10. De Grandpré, Jean-Paul: "Vocabulaire des assurances sociales ", vol. 12, no 1, p. 16. « Faut-il réclamer?", vol. 12, no 2, p. 51.

"La langue des assurances", vol. 12, $\mathrm{n}^{\circ} 4$, p. 133.

11. Desjardins, Philippe: "Deuxième biennale de la Fédération du français universel ", vol. $12, \mathrm{n}^{\circ} 2$, p. 71 .

12. Desjardins, Suzanne : "Terminologie pharmaceutique », vol. 12, n० 4, p. 130.

13. Dubuc, Robert: "Le français face à la civilisation scientifique et technique ", vol. 12, $\mathrm{n}^{\circ} 1$, p. 23.

“Les indésirables", vol. $12, \mathrm{n}^{\circ} 2$, p. 50.

"Ordinateur contre traducteurs", vol. 12, n $^{\circ} 2$, p. 70.

"La Société française des traducteurs a vingt ans », vol. 12, n 2, p. 70.

"Le jargon des sciences ", vol. $12, \mathrm{n}^{\circ} 3$, p. 94.

"Comité d'étude des termes techniques français ", vol. 12, n० 3, p. 97.

“Deuxième biennale de la langue française », vol. $12, n^{\circ} 4$, p. 143.

"Répertoire des termes et expressions utilisés en relations industrielles et dans des domaines connexes , vol. $12, \mathrm{n}^{\circ} 4, \mathrm{p} .137$.

14. Giraud, Jean : "Terminologie du cinéma ", vol. $12, \mathrm{n}^{\circ} 4, \mathrm{p} .118$

15. Grebenschikov, Vladimir : "La traduction en URSS ", vol. 12, no 1, p. 3.

16. Hanna, Blake T. : "Random House Dictionary of the English Language ", vol. 12, $\mathrm{n}^{\circ} 1$, p. 16.

"The Lexicon of the Sports and Racing Car Enthusiast", vol. 12, n० 2, p. 59.

"Hypnotism through Interpreter ", vol. 12, n 4, p. 142.

"International Center for Research on Bilingualism ", vol. 12, no 4, p. 141. 
17. Haumont, Roland: "Pensée, culture et message », vol. $12, n^{\circ} 3$, p. 75.

18. Henry, Anita : "Prix Paul Claudel ", vol. 12, n० 2, p. 72.

19. Horguelin, Paul A. : "Terminologie comptable», vol 12, no 1 , p. 17.

"Bibliographie ", vol. 12, n 1, p. 18.

"Catalogue des livres canadiens en librairie ", vol. 12, n 2, p. 60.

" Bibliographie ", vol. 12, nº 2, p. 61.

"Il y a de la marge ! ", vol. $12, \mathrm{n}^{\circ} 3$, p. 92.

"Bibliographie ", vol. 12, no 3, p. 98.

20. Lambert-Carey, Gérard: «Terminologie de l'informatique », vol. 12, no 4, p. 125.

21. Longpré, Marcel : "Vocabulaire de l'électricien", vol. 12, $\mathrm{n}^{\circ} 3$, p. 85 .

22. Meloche, Paul : "L'ATIO au cours de l'automne", vol. 12 , $\mathrm{n}^{\circ} 1$, p. 31 .

"Assemblée générale annuelle de 1'ATIO ", vol. 12, no 2, p. 68.

"L'ATIO sous un nouveau jour ", vol. $12, \mathrm{n}^{\circ} 3$, p. 106.

23. Nilski, Thérèse: "Translators and Interpreters", vol. 12, no 2, p. 45.

24. Paré, Marcel : "Vocabulaire de la langue des assurances sociales et des assemblées délibérantes ", vol. 12, $\mathrm{n}^{\circ} 3$, p. 97.

25. Plaice, Mary: "Société des Traducteurs du Québec", vol. 12, n 2, p. 69.

26. Pollet, Ray J. : "A Queer Trade ", vol. 12, no 3, p. 81.

27. Rosseel, Daniel : "Encore la machine à traduire", vol. 12, no 2, p. 71.

"Colloque sur la traduction automatique", vol. 12, no 3, p. 103.

28. Schmit, Claude : "En aluminium - d'aluminium ", vol. 12, no 2, p. 56. «Disbursement ", vol. $12, \mathrm{n}^{\circ} 3$, p. 88 .

29. Shapiro, Arthur: "Terminologie de l'informatique ", vol. 12, no 4, p. 125.

30. Walther, Ferdinand: "Société des Traducteurs du Québec ", vol. 12, no 1, p. 31. «Société des Traducteurs du Québec ", vol. 12, n 2, p. 69.

\section{B. ARTICLES}

« Brève réflexion sur la terminologie comptable $": 8$.

«Culture Vulture ": 4

«Défini, indéfini $»: 3$.

"Dossier (au) de " nursing *: 1.

"Faut-il réclamer?": 10.

«Il y a de la marge ! ": 19.

"Indésirables (les) ": 13

"Langue (la) des assurances " 10.

“Pensée, culture et message »: 17.

"Queer (A) Trade »: 26.

"Ressources néologiques du français contemporain $\approx: 9$.

"Table ronde sur la formation du traducteur $": 2$.

"Terminologie comptable ": 19.

"Terminologie de l'informatique ": 20, 29.

«Terminologie du cinéma *: 14.

"Terminologie pharmaceutique ": 12.

"Traduction (la) en URSS * : 15.

"Translators and Interpreters $:: 23$

"Vocabulaire de l'électricien »: 21

\section{CHRONIQUES - NEWS}

"Assemblée générale annuelle de l'ATIO ": 22.

"Association des Traducteurs et Interprètes de l'Ontario $»: 2$.

"ATIO (l') au cours de l'automne »: 22

" ATIO (l') sous un nouveau jour »: 22.

" Bureau de traduction du Nouveau-Brunswick $\gg$ : vol. 12 , no 4 , p. 142

"Centre de documentation technique ": vol 12 , no 2 , p. 60 .

« Cercle des Traducteurs ": $\mathbf{5 , 7 .}$
"Chronique de la STIC " 2.

"Colloque des diplômés de l'Université de Montréal »: vol, $12, \mathrm{n}^{\circ} 3$, p. 103

"Colloque sur la traduction automatique »: $\mathbf{2}$, 27.

"Colloque sur le bilinguisme »: vol. $12, \mathrm{n}^{\circ} 1$, p. 34.

"Comité d'étude des termes techniques francais $»: 13$.

"Corporation des Traducteurs professionnels du Québec »: vol. 12, n 2 , p. 69.

"Deuxième biennale de la Fédération du français universel »: 11.

"Deuxième biennale de la langue française ": 13.

" École d'interprètes et de traducteurs d'Ottawa ": vol. $12, \mathrm{n}^{\circ} 1$, p. 34 .

"Encore la machine à traduire »: 27.

" Français (le) face à la civilisation scientifique et technique $\gg: 2,13$.

"Hypnotism through Interpreter »: 16.

"International Center for Research on Bilingualism » : 16.

"Japan Society of Translators " : vol. 12, $\mathrm{n}^{\circ} 4$, p. 142.

«Ordinateur contre traducteurs »: 13.

«Prix Paul Claudel »: 18.

* Socićté des Traducteurs et Interprètes du Canada \#: 2.

"Société des Traducteurs du Québec »: 2, 25, 30, vol. $12, \mathrm{n}^{\circ} 3$, p. 106 .

"Société (la) française des traducteurs a vingt ans $: 13$.

\section{DIVERS - MISCELLANEOUS}

«A propos de « giggle»: 3. 
"Consultation ": 6.

\& Disbursement $»: 28$

* Documentation *: vol. $12, \mathrm{n}^{\circ} 3$, p. 96

* En aluminium - d'aluminium »:28.

* Traduction des livrets d'opéra ": vol. 12, no 3 , p. 102.

\section{E. COMPTES RENDUS - REVIEWS}

- Catalogue des livres canadiens en librairie» : 19.

« Bibliographie » 19.

« Jargon (le) des sciences $»: 13$.
"Lexicon (The) of the Sports and Racing Car Enthusiast ": 16.

"Portrait du vocabulaire français ": 9.

"Random House Dictionary of the English Language ": 16.

"Répertoire des termes et expressions utilisés en relations industrielles et dans des domaines connexes ": 13

"Structure et information ": 9.

* Vocabulaire de la langue des assurances sociales et des assemblées délibérantes ": 24.

"Vocabulaire des assurances sociales ": $\mathbf{1 0}$. 